\title{
All-Organic Textile Thermoelectrics with Carbon-Nanotube-Coated n-Type Yarns
}

\author{
Jason D. Ryan, ${ }^{\dagger}$ Anja Lund, ${ }^{\dagger}$ Anna I. Hofmann, ${ }^{\dagger}$ Renee Kroon, ${ }^{\dagger}$ Ruben Sarabia-Riquelme, ${ }^{\dagger}$ \\ Matthew C. Weisenberger, \\ ${ }^{\dagger}$ Department of Chemistry and Chemical Engineering, Chalmers University of Technology, 41296 Göteborg, Sweden \\ ${ }^{\ddagger}$ Department of Applied Energy Research, University of Kentucky, 2540 Research Park Drive, Lexington, Kentucky 40511, United \\ States
}

Supporting Information

ABSTRACT: Thermoelectric textiles that are able to generate electricity from heat gradients may find use as power sources for a wide range of miniature wearable electronics. To realize such thermoelectric textiles, both $\mathrm{p}$ - and n-type yarns are needed. The realization of air-stable and flexible n-type yarns, i.e., conducting yarns where electrons are the majority charge carriers, presents a considerable challenge due to the scarcity of air-stable n-doped organic materials. Here, we realize such n-type yarns by coating commercial sewing threads with a nanocomposite of multiwalled carbon nanotubes (MWNTs) and poly $(\mathrm{N}$ vinylpyrrolidone) (PVP). Our n-type yarns have a bulk conductivity of $1 \mathrm{~S} \mathrm{~cm}^{-1}$ and a Seebeck coefficient of $-14 \mu \mathrm{V} \mathrm{K} \mathrm{K}^{-1}$, which is stable for several months at ambient conditions. We combine our coated n-type yarns with poly $(3,4$ ethylenedioxythiophene):poly(styrenesulfonate) (PEDOT:PSS) dyed silk yarns, constituting the p-type component, to realize a textile thermoelectric module with $38 \mathrm{n} / \mathrm{p}$ elements, which are capable of producing an open-circuit voltage of $143 \mathrm{mV}$ when exposed to a temperature gradient of $116^{\circ} \mathrm{C}$ and a maximum power output of $7.1 \mathrm{nW}$ at a temperature gradient of $80{ }^{\circ} \mathrm{C}$.

KEYWORDS: organic thermoelectrics, carbon nanotubes, polymer nanocomposite, n-type conducting yarn, electronic textile

\section{INTRODUCTION}

Textiles are an intriguing platform for flexible electronics that may find use for a wide range of applications such as interactive garments for medical care and functional fabrics for climate control. To expand the reach of electronic textiles (e-textiles), it is desirable to develop truly autonomous systems that are able to generate power in situ and hence do not rely on batteries. A variety of power generating textiles that use the photovoltaic, ${ }^{1-4}$ piezoelectric, ${ }^{5,6}$ triboelectric, ${ }^{2,7-9}$ or thermoelectric effect $^{10-13}$ have been developed. We here focus on thermoelectric textiles, which would allow us to harvest electricity from temperature gradients such as the difference between a wearer's body temperature and the (typically colder) surroundings. Alternatively, such textiles could be used for on-the-spot cooling, e.g., when incorporated into sportswear, office chairs, or car seats.

To construct thermoelectric textiles from the bottom up, as a first step it is necessary to develop electrically conducting fibers or yarns. ${ }^{14}$ We argue that truly scalable solutions demand the use of organic materials, including polymers and carbon allotropes, which readily allow to adjust the mechanical and electrical properties of the conducting yarns, and currently receive renewed attention as thermoelectric materials. ${ }^{15-17}$ Suitable conducting yarns can then be incorporated into various textile architectures via weaving, knitting or stitching to form the basic unit of a thermoelectric generator: the thermoelectric leg.

When exposed to a temperature gradient, $\Delta T=T_{\text {hot }}-T_{\text {cold }}$ a single thermoelectric leg generates an electrical potential $\Delta V$ whose magnitude depends on the so-called Seebeck coefficient $\alpha$, a material specific parameter, according to

$$
\Delta V=-\alpha \Delta T
$$

A thermoelectric material can be either p- or n-type, i.e., their majority charge carriers are either holes or electrons, giving rise to a positive $\left(\alpha_{\mathrm{p}}>0\right)$ or negative $\left(\alpha_{n}<0\right)$ Seebeck coefficient. The generated electrical potential of a single leg is typically on the order of microvolts to millivolts, which is too small to be of practical use; most electronic components require at least one volt to operate. Therefore, it is necessary to connect many legs electrically in series but thermally in parallel, in order to create a configuration where individual thermovoltages add up to a sufficiently large output voltage. Pairs of adjacent legs form a thermoelectric element and the output voltage of the complete thermoelectric generator is given by

$$
\Delta V=N\left(\alpha_{1}-\alpha_{2}\right) \Delta T
$$

Received: April 18, 2018

Accepted: May 25, 2018

Published: June 11, 2018 
where $N$ is the total number of elements, and $\alpha_{1}$ and $\alpha_{2}$ are the Seebeck coefficients of the two legs of each element (Figure 1).

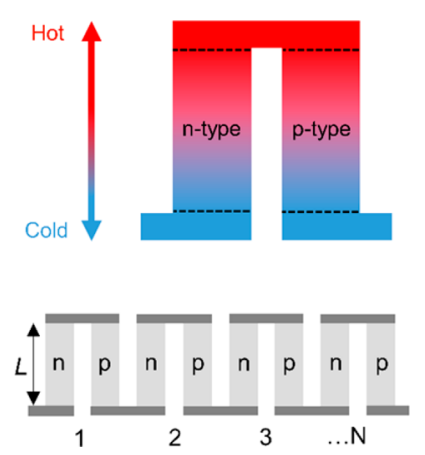

Figure 1. Schematic of a single thermoelectric element, and a row of $N$ elements thermally connected in parallel and electrically connected in series.

Ideally, an element comprises a p-type and a n-type leg so that individual voltages add, i.e., $\alpha_{1}=\alpha_{\mathrm{p}}$ and $\alpha_{2}=\alpha_{n}$ are of opposing sign. Therefore, to construct thermoelectric textiles both p-type and n-type yarns are needed.

A few examples of organic p-type yarns or textiles have been reported. ${ }^{12,13,18,19}$ For instance, we have prepared p-type yarns by dyeing silk from the silkworm Bombyx mori with the conjugated polymer:polyelectrolyte complex poly $(3,4-$ ethylenedioxythiophene):poly(styrenesulfonate) (PEDOT:PSS). ${ }^{13}$ The resulting conducting yarns featured a bulk electrical conductivity of $14 \mathrm{~S} \mathrm{~cm}^{-1}$ and a Seebeck coefficient of $+15 \mu \mathrm{V} \mathrm{K}^{-1}$, as well as excellent wash and wear resistance. In contrast, to date, reports on n-type fibers and yarns based on organic semiconductors are rare, which we ascribe to the lack of air-stable n-type organic semiconductors. A notable exception is the work by Ito et al., who demonstrated fibers with switchable $\mathrm{n}$ - and p-type behavior via selective $\mathrm{n}$-doping of wet-spun carbon nanotube fibers with 1-butyl-3-methylimidazolium hexafluorophosphate. ${ }^{20}$ In the absence of readily available ntype fibers we, as well as others, ${ }^{12,13,18,21,22}$ have explored the construction of prototype thermoelectric modules by electrically shorting p-type legs, e.g. comprised of PEDOT:PSS dyed silk, ${ }^{13}$ with metal (e.g., silver) films or wires. Silver features a positive but small Seebeck coefficient of $+1.5 \mu \mathrm{V} \mathrm{K}{ }^{-1}$, which according to eq 2 results in devices with a slightly reduced thermovoltage of $\Delta V=N\left(15 \mu \mathrm{V} \mathrm{K} \mathrm{K}^{-1}-1.5 \mu \mathrm{V} \mathrm{K} \mathrm{K}^{-1}\right) \Delta T$. Evidently, to further enhance the prospect of thermoelectric textiles it will be important to develop n-type yarns.

Here, we demonstrate air-stable organic n-type yarns that we fabricate by coating commercial poly(ethylene terephthalate) (PET) sewing threads with a nanocomposite of multiwalled carbon nanotubes (MWNTs) and poly $(\mathrm{N}$-vinylpyrrolidone) (PVP). We chose to work with this type of nanocomposite inspired by the recent work of Sarabia-Riquelme et al., who reported that MWNT:PVP nanocomposites readily display airstable n-type behavior. ${ }^{23}$ Besides PVP, a number of other polymers such as poly(ethylenimine) (PEI), ${ }^{24,25}$ poly(vinyl alcohol) (PVA), ${ }^{26}$ poly(vinylidene fluoride) (PVDF), ${ }^{27}$ poly(ethylene glycol) (PEG), ${ }^{28}$ and poly(3-hexylthiophene) $(\mathrm{P} 3 \mathrm{HT})^{29}$ have been found to change the type of majority charge carriers of (oxygen doped) carbon nanotubes from holes to electrons, resulting in a negative Seebeck coefficient. A further attractive feature of the nanocomposite approach is that, as a surrounding polymer matrix has been shown to mitigate the release of carbon nanofillers, ${ }^{30,31}$ the use of a composite rather than neat $\mathrm{CNTs}$ as a coating should reduce exposure to the potentially toxic CNTs. To further improve the wear and water resistance of our coated yarns, we equipped them with an outer layer of a polystyrene- $b$-polyisoprene- $b$-polystyrene block copolymer (SIS). Our coated PET yarns feature an air-stable and negative Seebeck coefficient of $-14 \mu \mathrm{V} \mathrm{K}^{-1}$ and a bulk conductivity of about $1 \mathrm{~S} \mathrm{~cm}^{-1}$. To showcase the properties of the here developed n-type yarns, we demonstrate an all-organic thermoelectric textile consisting of 38 embroidered elements with an output voltage of $143 \mathrm{mV}$ for a $\Delta T \approx 116^{\circ} \mathrm{C}$.

\section{RESULTS AND DISCUSSION}

To realize conducting n-type yarns, we chose to work with a MWNT:PVP nanocomposite that is known to feature a negative Seebeck coefficient. ${ }^{23}$ PET yarns consisting of about 100 filaments were coated with the MWNT:PVP nanocomposite using a three-step coating process (Figure 2), similar

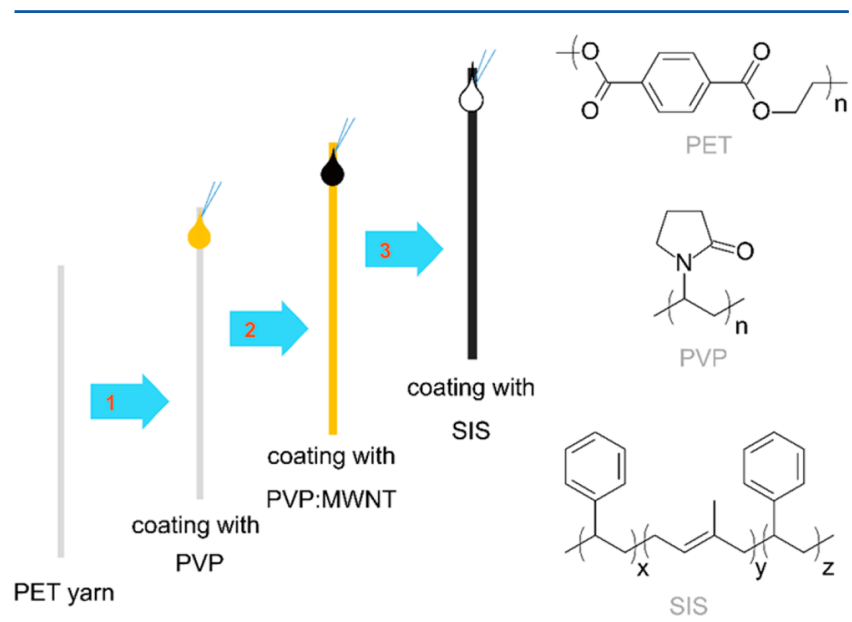

Figure 2. Schematic representation of the three step coating process of PET yarn with (1) PVP adhesion layer, (2) MWNT:PVP conducting layer, and (3) outer SIS protection layer; chemical structures of PET, PVP, and SIS.

to the method presented by Steven et al. ${ }^{32}$ To improve the wetting of the MWNT:PVP solution on the PET yarn, we first coated the pristine yarn with an adhesion layer using a highly concentrated PVP solution (500 $\mathrm{g} \mathrm{L}^{-1}$ ) (see Figure S1 and Experimental Section for details). This PVP coating considerably improved the adhesion of the subsequent conducting layer, which we applied by coating with a $150 \mathrm{~g} \mathrm{~L}^{-1}$ aqueous solution of 1:4 MWNT:PVP by weight. We chose to work with a highly concentrated solution in order to obtain a sufficiently high solution viscosity, which was needed for coating of PET yarns. We also explored the addition of $1 \mathrm{~g} \mathrm{~L}^{-1}$ of PEI, which has been found to result in a more negative Seebeck coefficient. $^{23}$ Finally, we applied an elastomeric protection layer consisting of SIS, which afforded a two-way protection of the yarn from abrasion and damage, as well as the user from possible carbon nanotube exposure. Optical microscopy and scanning electron microscopy (SEM) images both suggest that the PVP and MWNT:PVP coatings penetrate the yarn and to some extent coat the individual PET fibers, thus retaining the surface topography of the yarn (Figure $3 a, b, d$ ). The SIS coating layer, finally, wraps the outside of the yarn resulting in a smooth and continuous appearance (Figure $3 c-e$ and Figure S2). 


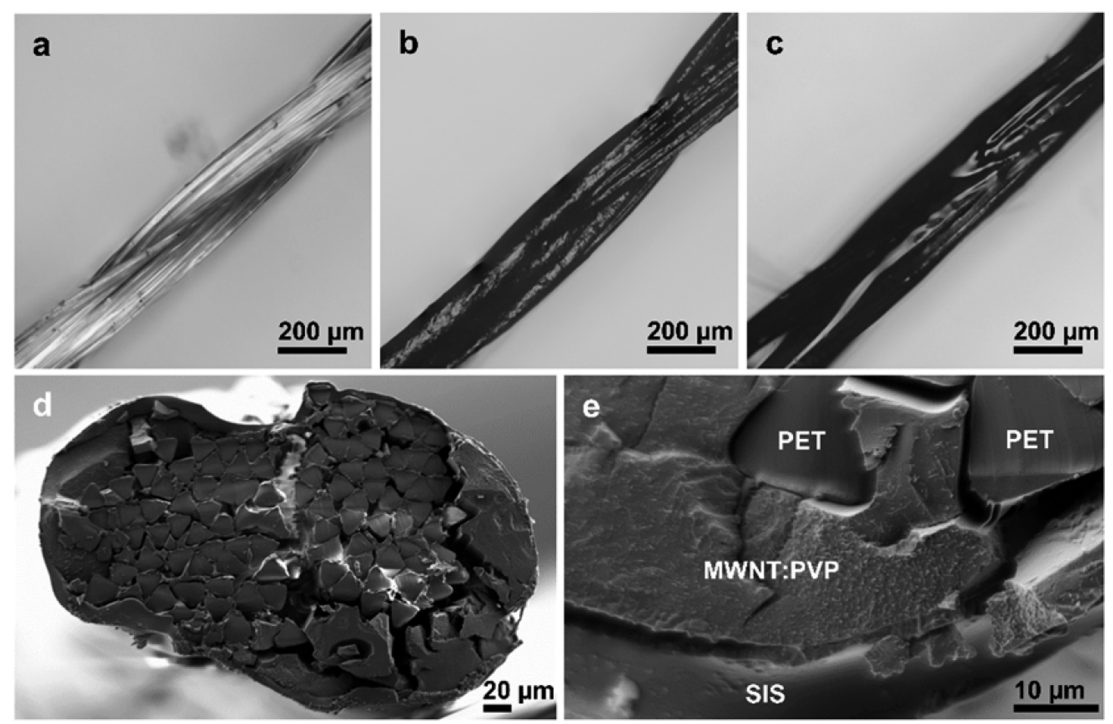

Figure 3. Optical microscopy images of: (a) neat PET yarn; (b) PET yarn coated with a PVP adhesion layer and MWNT:PVP conducting layer; (c) PET yarn coated with PVP, MWNT:PVP, and a SIS protection layer (d) SEM image of the cross-section of a cut PET yarn coated with PVP, MWNT:PVP, and SIS ; and (e) SEM image, close up of the same yarn showing the individual PET fibers embedded in the MWNT:PVP coating, surrounded by the outermost SIS coating.; and (e) SEM image, close up of the same yarn showing the individual PET fibers embedded in the MWNT:PVP coating, surrounded by the outermost SIS coating.

The thermoelectric properties of the here prepared n-type yarns notably improve during the first 4 days after coating, which we attribute to further drying of the coating formulation, whereafter both the Seebeck coefficient and the electrical conductivity level out (Figure 4). Four days after coating the yarns featured a bulk electrical conductivity of $\sigma_{n} \approx 1 \mathrm{~S} \mathrm{~cm}^{-1}$ and a Seebeck coefficient of $\alpha_{\mathrm{n}} \approx-14 \mu \mathrm{V} \mathrm{K}^{-1}$ (Table 1), which translates into a thermoelectric power factor of $\alpha_{\mathrm{n}}^{2} \sigma_{\mathrm{n}} \sim 10^{-2} \mu \mathrm{W}$ $\mathrm{m}^{-1} \mathrm{~K}^{-2}$. We do not observe a significant difference in thermoelectric properties when adding PEI to the conducting layer (cf. Table 1). Remarkably, the yarns display stable n-type behavior for at least six months under ambient conditions (from -11 to $-15 \mu \mathrm{V} \mathrm{K} \mathrm{K}^{-1}$ ). Analysis of the weight increase after each coating step suggests that $36 \%$ of the coated yarn is composed of the PVP adhesion layer, $18 \%$ is the MWNT:PVP nanocomposite layer, and $8 \%$ is the SIS protection layer. Taking into account that only the MWNT:PVP layer contributes to the electrical conductivity of the composite fiber, we can estimate that the conductivity of the MWNT:PVP composite amounts to approximately $5 \mathrm{~S} \mathrm{~cm}^{-1}$. We measured very similar values for drop cast films of MWNT:PVP, indicating that the yarn geometry does not negatively affect the thermoelectric properties (see Table S1).

In a further set of experiments, we studied the mechanical properties of the here prepared n-type yarns. We find that the coating process does not appear to influence the mechanical properties of the PET yarns. Both neat and coated yarns display a similar Young's modulus $E \approx 3 \mathrm{GPa}$ (Table 1 ). We conclude that the load bearing part of the n-type yarns is the nonconducting PET multifilament core. The mechanical robustness of yarns is paramount for both many textile manufacturing processes as well as for later wear resistance of the finished fabric. We therefore subjected n-type yarns to multiple bending cycles using an in-house designed LEGO setup (Figure 5a). We observe that the electrical resistance gradually increases with the number of bending cycles. Both ntype yarns without and with the outer SIS protection layer tend to display an about 1 order of magnitude increase in resistance after 1000 bending cycles (Figure $5 \mathrm{~b}$ ). We therefore conclude that one-off bending of the yarns during textile manufacturing is unlikely to drastically reduce the thermoelectric properties. However, in their current form the use of these yarns is only recommended in textiles where they are not subjected to repeated bending during manufacture and use. For instance, the yarns can be used for stitching and hand weaving, while knitted structures are not suitable. ${ }^{14}$ Moreover, the bending stability needs to be improved before the yarns can be expected to withstand machine-washing.

Besides a promising stability of the electrical and mechanical properties, we would like to highlight the water resistance of the here studied n-type yarns. The SIS outer protection layer is water repellent and can be thought of as a cable insulation with the PET yarn plus MWNT:PVP shell being the conducting core. We measured the electrical resistance of a fully coated yarn before, during and after submersion in water, and observed no notable increase in overall resistance (Figure 5c). Evidently, our n-type yarns are sufficiently inert to be used under practical conditions, i.e., in a textile that comes in contact with water.

We went on to construct prototype thermoelectric modules with MWNT:PVP n-type yarns in order to explore their suitability for e-textile applications. For the p-type yarns we used PEDOT:PSS dyed silk, which we have reported previously. ${ }^{13}$ As compared to the here developed n-type yarns, our p-type yarns display a similar absolute but positive Seebeck coefficient of $\alpha_{\mathrm{p}} \approx+14 \mu \mathrm{V} \mathrm{K} \mathrm{K}^{-1}$ but 1 order of magnitude higher electrical conductivity of $\sigma_{\mathrm{p}} \approx 15 \mathrm{~S} \mathrm{~cm}^{-1}$. We stitched the conducting yarns onto wool swatches, using couch stitch. Initially, we fabricated a small in-plane device that consisted of four $\mathrm{n} / \mathrm{p}$ elements with a total internal resistance of $R_{\text {in }} \approx 34 \mathrm{k} \Omega$. In order to reduce $R_{\text {in }}$ we used $11 \mathrm{n}$-type and two $\mathrm{p}$-type yarns, respectively (Figure $6 \mathrm{a}$ ). We connected individual $7 \mathrm{~cm}$ long legs with flexible silver paste over a length of $1 \mathrm{~cm}$ at both ends. We then placed one end of the device on a hot plate $\left(T_{\text {hot }} \leq 105{ }^{\circ} \mathrm{C}\right)$ and the other end on a cold plate $\left(T_{\text {cold }} \approx 25\right.$ ${ }^{\circ} \mathrm{C}$ ) to establish a temperature gradient of $\Delta T \leq 80{ }^{\circ} \mathrm{C}$ as measured with two thermocouples. The output voltage of the 


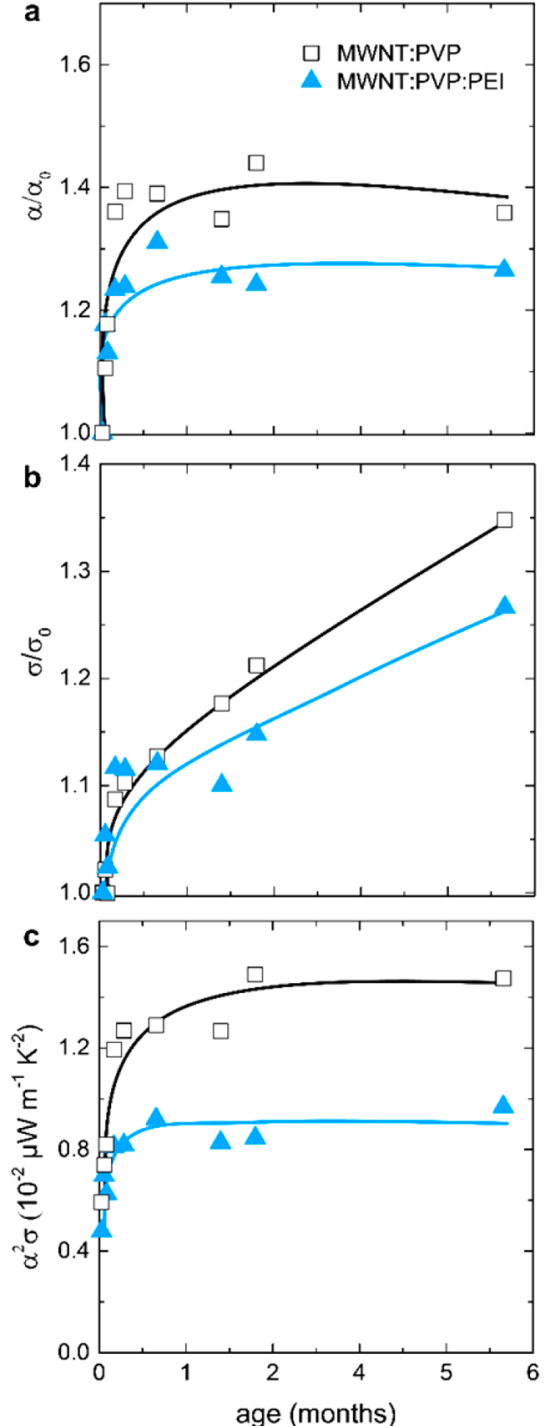

Figure 4. Aging of the thermoelectric properties of MWNT:PVP coated PET yarns under ambient conditions: (a) Seebeck coefficient $\left(\alpha_{0} \approx-11 \mu \mathrm{V} \mathrm{K}^{-1}\right),(\mathrm{b})$ conductivity $\left(\sigma_{0} \approx 0.5 \mathrm{~S} \mathrm{~cm} \mathrm{~cm}^{-1}\right)$, and (c) power factor $\alpha^{2} \sigma$ (open squares, MWNT:PVP, blue triangles, MWNT:PVP:PEI). Note that although the conductivity in $b$ apparently increases over time, the observed change lies within the error of the measurement, see Table 1 .

device increased linearly with $\Delta T$ and reached a value of $V_{\text {out }} \approx$ $8 \mathrm{mV}$ for $\Delta T \approx 80{ }^{\circ} \mathrm{C}$ (Figure $6 \mathrm{~b}$ ). We can estimate the relative output voltage per temperature difference $V_{\text {out }} / \Delta T=N\left(\alpha_{\mathrm{p}}\right.$ a

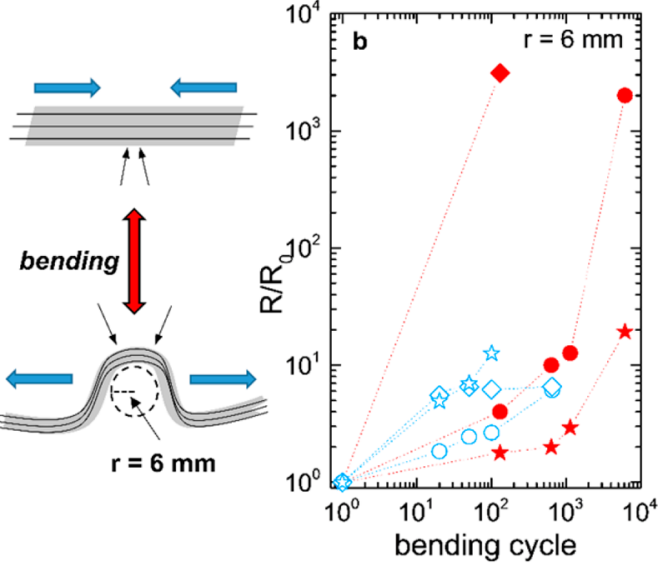

c
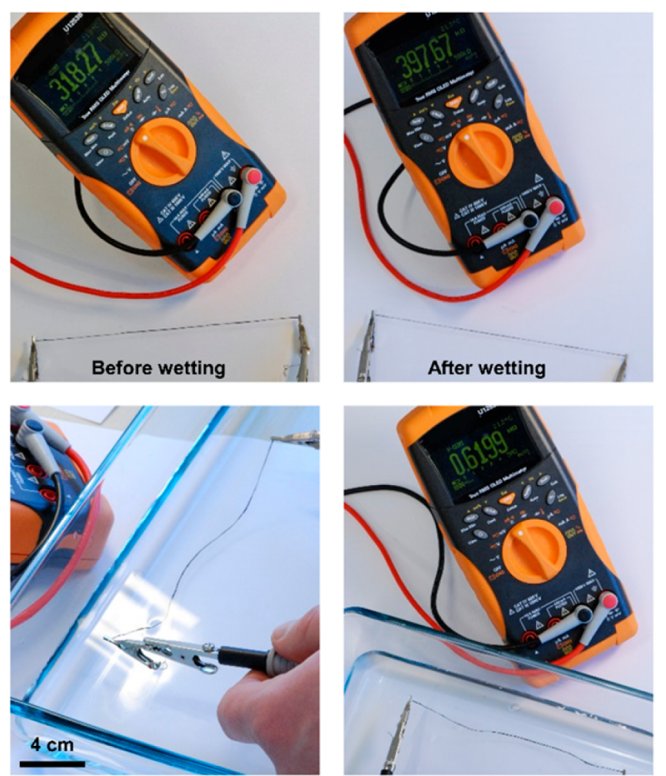

Figure 5. (a) Schematic diagram of the bending test performed with a LEGO setup; (b) resistance $R$ of $\sim 7 \mathrm{~cm}$ long yarns bent repeatedly along with a PET foil support (different symbols denote different yarn samples, filled and empty symbols represent fibers with and without SIS coating, respectively); (c) photographs of resistance measurements of MWNT:PVP + SIS coated PET yarn before water submersion (top left), during water submersion (bottom left and bottom right) and after submersion (top right).

$\left.\alpha_{\mathrm{n}}\right) \approx 116 \mu \mathrm{V} \mathrm{K} \mathrm{K}^{-1}$ according to eq 2 , which is in good agreement with the experimentally obtained value of $104 \mu \mathrm{V}$ $\mathrm{K}^{-1}$ that corresponds to the slope in Figure $6 \mathrm{~b}$. We also determined the maximum power output of the device at $\Delta T \approx$

Table 1. Mechanical and Electrical Properties of PET Yarns Precoated with PVP, then Coated with MWNT:PVP, and Finally SIS: Stress at Break $S_{\mathrm{b}}$, Strain at Break $\varepsilon_{\mathrm{b}}$, Young's Modulus E, Electrical Conductivity $\sigma_{n}$ of the Bulk Fiber, Seebeck Coefficient $\alpha_{n}$, Number of Samples Measured, $n^{a}$

\begin{tabular}{|c|c|c|c|c|c|c|c|c|c|c|}
\hline \multirow[b]{2}{*}{ yarn } & \multirow[b]{2}{*}{ polymer matrix } & \multirow[b]{2}{*}{ protective coating } & \multicolumn{4}{|c|}{ mechanical properties } & \multicolumn{4}{|c|}{ electrical properties } \\
\hline & & & $S_{\mathrm{b}}(\mathrm{MPa})$ & $\varepsilon_{\mathrm{b}}(\%)$ & $E(\mathrm{GPa})$ & $n$ & $\sigma_{n}\left(\mathrm{~S} \mathrm{~cm}^{-1}\right)$ & $n$ & $\alpha_{n}\left(\mu \mathrm{V} \mathrm{K}^{-1}\right)$ & $n$ \\
\hline \multirow[t]{5}{*}{ PET } & & & $375 \pm 18$ & $37 \pm 8$ & $2.7 \pm 0.4$ & 3 & $b$ & & $b$ & \\
\hline & PVP & & $c$ & & & & $0.8 \pm 0.2$ & 5 & $-12 \pm 1$ & 3 \\
\hline & PVP & SIS & $356 \pm 48$ & $26 \pm 6$ & $3.6 \pm 1.0$ & 5 & $0.8 \pm 0.2$ & 5 & $-14 \pm 1$ & 3 \\
\hline & PVP:PEI & & $c$ & & & & $1.0 \pm 0.3$ & 9 & $-14 \pm 1$ & 3 \\
\hline & PVP:PEI & SIS & $386 \pm 14$ & $32 \pm 2$ & $2.8 \pm 0.8$ & 5 & $0.7 \pm 0.2$ & 5 & $-15 \pm 1$ & 3 \\
\hline
\end{tabular}

${ }^{a}$ All measurements were made 4 days after fabrication. ${ }^{b}$ Nonconducting. ${ }^{c}$ Too delicate coating for tensile drawing. 

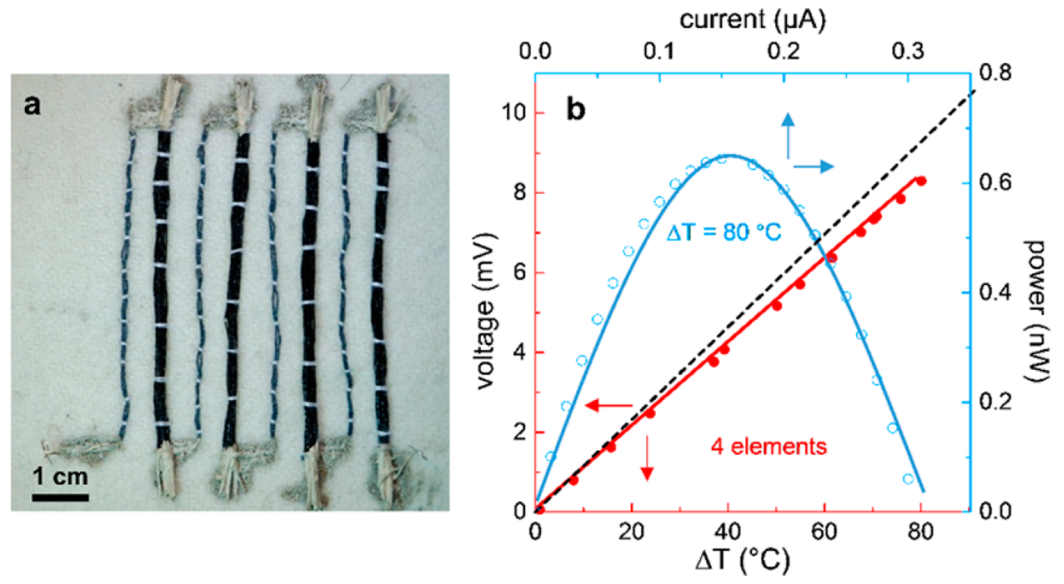

Figure 6. (a) In-plane embroidered textile thermoelectric device with $4 \mathrm{n} / \mathrm{p}$ elements, comprised of $\mathrm{n}$-type coated PET yarns ( 11 yarns per leg), ptype dyed silk yarns ( 2 yarns per leg) and silver paste for contacts; (b) electrical measurements of the module with measured output voltage $V_{\text {out }}$ as a function of $\Delta T$ (red line), and calculated according to eq 2 (dotted line), as well as power output $P=V_{\text {out }} I$ as a function of measured current $I$ for $\Delta T \approx 80^{\circ} \mathrm{C}$.
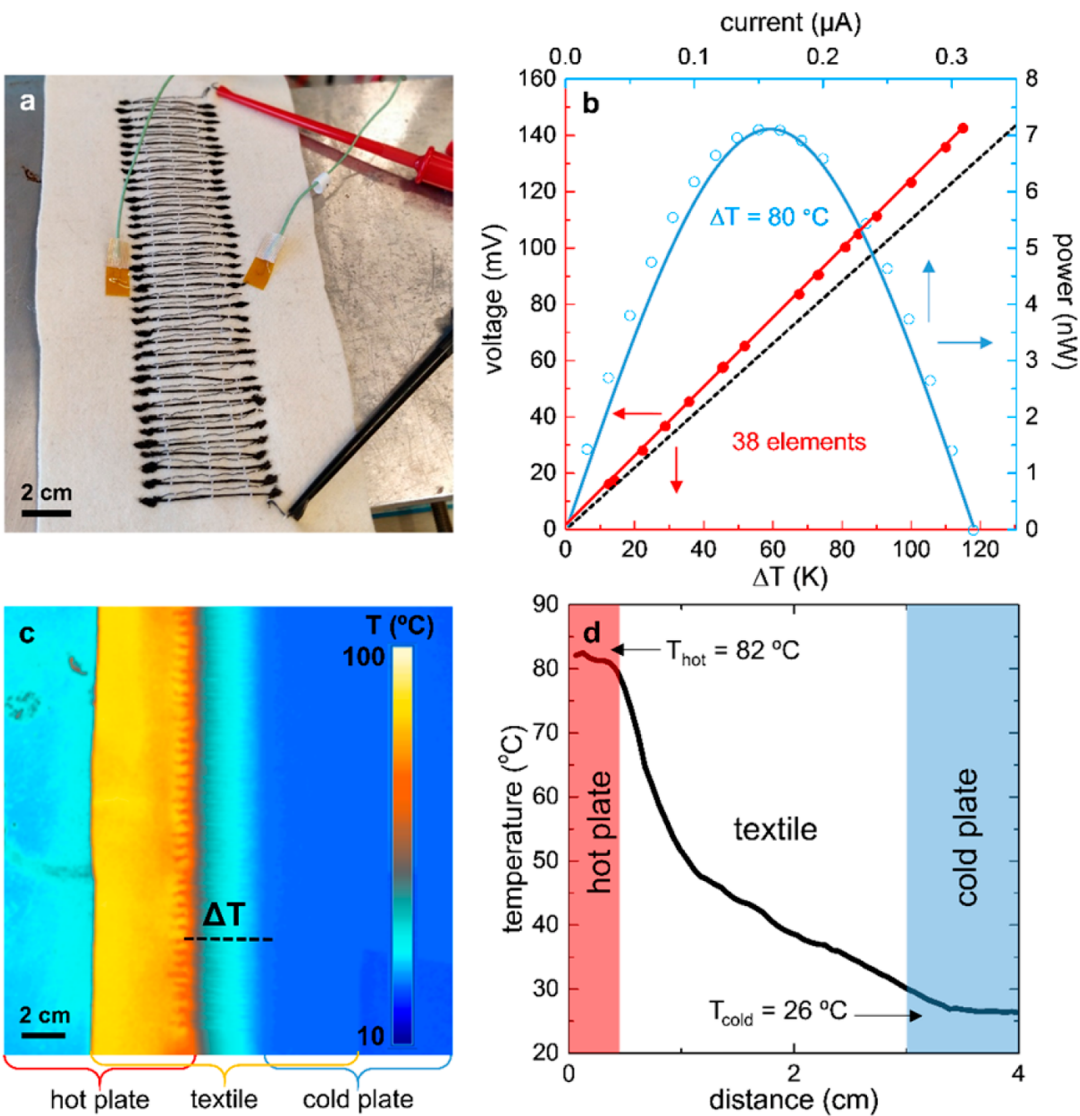

Figure 7. (a) All-organic in-plane embroidered textile thermoelectric device with $38 \mathrm{n} / \mathrm{p}$ elements-constructed with n-type coated PET yarns (3 yarns per leg), p-type dyed silk yarns ( 1 yarn per leg) and a conducting carbon-based paste for electrical connections- and suspended between a hot and cold plate, with two adhesive thermocouples on top; (b) electrical measurements of the module with measured output voltage $V_{\text {out }}$ as a function of $\Delta T$ (red line), and calculated according to eq 2 (dotted line), as well as power output $P=V_{\text {out }} I$ as a function of measured current $I$ for $\Delta T \approx 80$ ${ }^{\circ} \mathrm{C}$; (c) thermal image of the module, placed as in a, with $T_{\text {hot }} \approx 82{ }^{\circ} \mathrm{C}$ and $T_{\text {cold }} \approx 26{ }^{\circ} \mathrm{C}$ (note that in the image the bare hot plate appears to be cold, due to the relatively low emissivity of aluminum); (d) temperature gradient across the textile device (see dashed line in $\mathrm{c}$ ), $T_{\text {hot }}$ and $T_{\text {cold }}$ measured with thermocouples (red box $=$ hot plate; blue box $=$ cold plate).

$80{ }^{\circ} \mathrm{C}$ for a variety of load currents. We obtain a maximum power output of $P_{\max } \approx 0.65 \mathrm{nW}$. The maximum power output can be calculated according to

$$
P_{\max }=V_{\text {out }}^{2} / 4 R_{\text {in }}
$$

yielding $P_{\max } \approx 0.49 \mathrm{nW}$, which is in near agreement with the experimentally obtained value.

Finally, we prepared a scaled up thermoelectric textile module with 38 elements (Figure 7a), this time with $4 \mathrm{~cm}$ long legs. We designed this all-organic module to be more compact 
and space efficient, and used 3 yarns per n-type leg and 1 yarn per p-type leg, which is close to the ratio required to maximize the power that is generated by a module with a certain total area and area coverage (see the Supporting Information). The whole n-type yarn was coated with SIS, with the ends cut to reveal the conducting coating. The exposed ends were connected to the p-type yarn with a commercial carboncontaining conducting paste developed for textile coatings. Conducting silk yarn, together with a small amount of the conducting paste, was used as the connections to the testing setup. Then, one side of the textile module was placed on a hot plate and the other side on a cold plate (cf. Figure $7 \mathrm{a}$ ). We applied a temperature difference of up to $\Delta T \sim 116{ }^{\circ} \mathrm{C}$ to achieve an open circuit voltage of $V_{\text {out }} \approx 143 \mathrm{mV}$, or $V_{\text {out }} / \Delta T \approx$ $1.23 \mathrm{mV} \mathrm{K}^{-1}$, which corresponds to about $32 \mu \mathrm{V} \mathrm{K}^{-1}$ per element (Figure $7 \mathrm{~b}$ ). The experimentally observed value slightly surpasses the predicted value of $V_{\text {out }} / \Delta T \approx 1.10 \mathrm{mV}$ $\mathrm{K}^{-1}$ (cf. eq 2). Finally, we determined the power for a range of load currents while holding the device at a temperature difference $\Delta T \approx 80^{\circ} \mathrm{C}$. We measured a value of $P_{\max } \sim 7.1 \mathrm{nW}$, which is in in good agreement with the predicted value of $P_{\max }$ $\approx 7.4 \mathrm{nW}$, calculated from an internal resistance of $R_{\mathrm{in}} \sim 334$ $\mathrm{k} \Omega$ (cf. eq 3). Thermal imaging revealed that a distinct temperature gradient is established parallel to the legs (Figure $7 \mathrm{c}$ ). Assuming a thermal emissivity of 0.95 we could extract the shape of the temperature gradient across the device (Figure $7 \mathrm{~d})$. We find that with increasing distance from the hot plate the temperature first sharply decreases with $\sim 6{ }^{\circ} \mathrm{C} \mathrm{mm}{ }^{-1}$ and after $5 \mathrm{~mm}$ with $\sim 1{ }^{\circ} \mathrm{C} \mathrm{mm}{ }^{-1}$, reaching a close to constant value at the edge of the cold plate.

\section{CONCLUSIONS}

In conclusion, we have created n-type yarns by coating PET yarns with a nanocomposite of multiwalled carbon nanotubes and poly $(N$-vinylpyrrolidone $)$. We used a layer of PVP to enhance adhesion of the conducting coating and improved the abrasion resistance by applying a protective coating consisting of a polystyrene- $b$-polyisoprene- $b$-polystyrene block copolymer. The n-type yarns displayed a sufficient degree of resilience to bending to permit stitching. We fabricated textile based thermoelectric modules that comprised MWNT:PVP coated n-type yarns and PEDOT:PSS dyed p-type yarns. An allorganic thermoelectric textile with $38 \mathrm{n} / \mathrm{p}$ elements generated an output voltage of $143 \mathrm{mV}$ when applying a temperature gradient of $116^{\circ} \mathrm{C}$, and a maximum power output of $7.1 \mathrm{nW}$ at a temperature gradient of $80{ }^{\circ} \mathrm{C}$. We anticipate that further improvement of $n$-type fibers and yarns in terms of electrical conductivity as well as wash and wear resistance will open up for the design of truly robust e-textiles with a wide range of functionalities.

\section{METHODS}

Materials. Commercial polyethylene terephthalate sewing threads were used without modification/pretreatment $(\varnothing \sim 0.19 \mathrm{~mm})$. Poly $(N$-vinylpyrrolidone $)$ was purchased from Polysciences $\left(M_{\mathrm{w}} \approx 40\right.$ $\left.\mathrm{kg} \mathrm{mol}^{-1}\right)$. Aligned multiwalled carbon nanotubes were CVD synthesized according to a previously published procedure. ${ }^{33}$ The particular MWNTs used here stemmed from a continuous and scaledup version of this process at the University of Kentucky, Center for Applied Energy Research. ${ }^{34,35}$ Polyethylenimine (branched, $M_{\mathrm{w}} \approx 25$ $\mathrm{kg} \mathrm{mol}^{-1}$ ), polystyrene- $b$-polyisoprene- $b$-polystyrene (styrene content 22 wt \%), dimethyl sulfoxide (DMSO) and toluene were purchased from Sigma-Aldrich and used as received. Silver paint was purchased from Agar Scientific, UK. For the small thermoelectric module, stretchable silver paste (PE872 Conductor paste, DuPont, UK) was used. After application the silver paste was dried at room temperature for $1 \mathrm{~h}$, then cured at $100{ }^{\circ} \mathrm{C}$ for $10 \mathrm{~min}$. Carbon-containing paste (TUBICOAT ELC, CHT, Germany) was used for the large module, dried at $80{ }^{\circ} \mathrm{C}$ for $12 \mathrm{~min}$ then cured at $140{ }^{\circ} \mathrm{C}$ for $2 \mathrm{~min}$.

Sample Preparation. MWNTs and PVP (ratio 1:4) were added to deionized water with final concentrations of 30 and $120 \mathrm{~g} \mathrm{~L}^{-1}$, respectively. Optionally, PEI ( 0.05 wt \% with regard to MWNTs) was added using a $1 \mathrm{~g} \mathrm{~L}^{-1}$ PEI solution to make up final concentrations stated. The carbon nanotube based solutions were ice bath sonicated for $\sim 40 \mathrm{~min}$ (power $=175 \mathrm{~W}$; Ultrasonik, NEY, USA), followed by 30 min active time probe sonication in pulse mode ( $5 \mathrm{~s}$ on, $5 \mathrm{~s}$ off, $20 \%$ amplitude, maximum power $=500 \mathrm{~W}$; Sonics and Materials Inc., USA). PET yarns were coated twice with PVP solution $\left(500 \mathrm{~g} \mathrm{~L}^{-1}\right)-$ to allow the PET to be wetted by MWNT:PVP solution - by dripping the solution down vertically hanging yarns, squeezing the yarn with (lab glove protected) fingers and running down the yarn to remove excess, and drying $30 \mathrm{~min}$ after each coating. The PVP coated PET yarns were coated by (lab glove protected) hand, with MWNT:PVP (or MWNT:PVP:PEI) solutions 7 times, with 10 min drying time after each layer. SIS copolymer in toluene $\left(100 \mathrm{~g} \mathrm{~L}^{-1}\right)$ was dripped twice along the coated PET yarn and yarn and excess polymer was carefully removed. PEDOT:PSS silk yarns were prepared according to a previously published procedure, ${ }^{13}$ using PEDOT:PSS aqueous dispersion from Heraeus, Germany to which 5 vol \% DMSO was added.

Light Microscopy. Light microscopy was carried out in bright field reflected light mode with a Carl Zeiss Al optical microscope.

Scanning Electron Microscopy. SEM images were taken with a Leo Ultra 55 SEM equipped with a field emission gun (LEO Electron Microscopy Group, Germany), utilizing the secondary electron detector. The acceleration voltage was $3 \mathrm{kV}$. Samples were frozen in liquid nitrogen prior to being cut with a scalpel, after which they were sputtered with palladium.

Mechanical Characterization. Tensile testing of $5 \mathrm{~cm}$ long yarns was carried out with an Instron tensile tester (model 5565A) at a cross-head speed of $10 \mathrm{~mm} \mathrm{~min}^{-1}$.

Electrical Characterization. The electrical resistivity of drop-cast films with a thickness of $\sim 0.3 \mathrm{~mm}$ was determined with a four-point probe setup from Jandel Engineering (cylindrical probe head, RM3000) using collinear tungsten carbide electrodes with $1 \mathrm{~mm}$ spacing. The electrical resistance of yarns was determined with a Keithley 2400 sourcemeter in two-point-probe configuration by contacting several $\sim 1 \mathrm{~cm}$ long segments with silver paint (see Table 1 for number of measured segments, $n$ ). The Seebeck coefficient of $\sim 5$ $\mathrm{mm}$ long segments was measured at $300 \mathrm{~K}$ with an SB1000 instrument equipped with a K2000 temperature controller from MMR Technologies using a thermal load of about $1-2 \mathrm{~K}$ and a constantan wire as an internal reference. Samples were mounted with silver paint. The resistance of yarns supported by PET films, during cyclic bending of radius $\sim 6 \mathrm{~mm}$, was measured with a Keithley 2400 sourcemeter using an in-house designed setup constructed with leg godt (LEGO). The thermoelectric modules were characterized utilizing a Keithley 2400 sourcemeter to measure voltage while-when characterizing generated power- simultaneously acting as a variable load by sinking current from the textile module. The modules were placed with one end on top of a programmable hot plate (Torrey Pines Scientific) and the other on an aluminum plate, which was held at room temperature. Two $\mathrm{K}$ type adhesive thermocouples (Omega Engineering) were placed on top of the wool fabric on the hot and cold sides, respectively.

Thermal Imaging. Thermal images were taken with a FLIR A645sc thermal camera and the thermal gradient graphs were derived using the accompanying software (ResearchIR 4).

\section{ASSOCIATED CONTENT}

\section{Supporting Information}

The Supporting Information is available free of charge on the ACS Publications website at DOI: 10.1021/acsaem.8b00617. 
Coating method, SEM images, thermoelectric properties, bending setup (PDF)

\section{AUTHOR INFORMATION}

\section{Corresponding Author}

*E-mail: christian.muller@chalmers.se.

\section{ORCID 1}

Christian Müller: 0000-0001-7859-7909

\section{Notes}

The authors declare no competing financial interest.

\section{ACKNOWLEDGMENTS}

Financial support from the Swedish Research Council Formas, the Knut and Alice Wallenberg Foundation through a Wallenberg Academy Fellowship, and the European Research Council (ERC) under grant agreement 637624 is gratefully acknowledged.

\section{REFERENCES}

(1) Weng, W.; Chen, P. N.; He, S. S.; Sun, X. M.; Peng, H. S. Smart Electronic Textiles. Angew. Chem., Int. Ed. 2016, 55, 6140-6169.

(2) Chen, J.; Huang, Y.; Zhang, N. N.; Zou, H. Y.; Liu, R. Y.; Tao, C. Y.; Fan, X.; Wang, Z. L. Micro-Cable Structured Textile for Simultaneously Harvesting Solar and Mechanical Energy. Nature Energy 2016, 1, 16138.

(3) Krebs, F. C.; Biancardo, M.; Winther-Jensen, B.; Spanggard, H.; Alstrup, J. Strategies for Incorporation of Polymer Photovoltaics Into Garments and Textiles. Sol. Energy Mater. Sol. Cells 2006, 90, 10581067.

(4) Zhang, N. N.; Chen, J.; Huang, Y.; Guo, W. W.; Yang, J.; Du, J.; Fan, X.; Tao, C. Y. A Wearable All-Solid Photovoltaic Textile. Adv. Mater. 2016, 28, 263-269.

(5) Soin, N.; Shah, T. H.; Anand, S. C.; Geng, J. F.; Pornwannachai, W.; Mandal, P.; Reid, D.; Sharma, S.; Hadimani, R. L.; Bayramol, D. V.; Siores, E. Novel "3-D Spacer" All Fibre Piezoelectric Textiles for Energy Harvesting Applications. Energy Environ. Sci. 2014, 7, 16701679.

(6) Lund, A.; Rundqvist, K.; Nilsson, E.; Yu, L.; Hagström, B.; Müller, C. Energy Harvesting Textiles for a Rainy Day: Woven Piezoelectrics Based on Melt-Spun PVDF Microfibres with a Conducting Core. npj Flex. Electron. 2018, 2, 9.

(7) Pu, X.; Li, L. X.; Song, H. Q.; Du, C. H.; Zhao, Z. F.; Jiang, C. Y.; Cao, G. Z.; Hu, W. G.; Wang, Z. L. A Self-Charging Power Unit by Integration of a Textile Triboelectric Nanogenerator and a Flexible Lithium-Ion Battery for Wearable Electronics. Adv. Mater. 2015, 27, 2472-2478.

(8) Liu, L. M.; Pan, J.; Chen, P. N.; Zhang, J.; Yu, X. H.; Ding, X.; Wang, B. J.; Sun, X. M.; Peng, H. S. A Triboelectric Textile Templated by a Three-Dimensionally Penetrated Fabric. J. Mater. Chem. A 2016, 4, 6077-6083.

(9) Seung, W.; Gupta, M. K.; Lee, K. Y.; Shin, K. S.; Lee, J. H.; Kim, T. Y.; Kim, S.; Lin, J.; Kim, J. H.; Kim, S. W. Nanopatterned TextileBased Wearable Triboelectric Nanogenerator. ACS Nano 2015, 9, 3501-3509.

(10) Lee, J. A.; Aliev, A. E.; Bykova, J. S.; de Andrade, M. J.; Kim, D.; Sim, H. J.; Lepro, X.; Zakhidov, A. A.; Lee, J. B.; Spinks, G. M.; Roth, S.; Kim, S. J.; Baughman, R. H. Woven-Yarn Thermoelectric Textiles. Adv. Mater. 2016, 28, 5038-5044.

(11) Kim, S. J.; We, J. H.; Cho, B. J. A Wearable Thermoelectric Generator Fabricated on a Glass Fabric. Energy Environ. Sci. 2014, 7, 1959-1965.

(12) Du, Y.; Cai, K. F.; Chen, S.; Wang, H. X.; Shen, S. Z.; Donelson, R; Lin, T. Thermoelectric Fabrics: Toward Power Generating Clothing. Sci. Rep. 2015, 5, 6411.
(13) Ryan, J. D.; Mengistie, D. A.; Gabrielsson, R.; Lund, A.; Müller, C. Machine-Washable PEDOT:PSS Dyed Silk Yarns for Electronic Textiles. ACS Appl. Mater. Interfaces 2017, 9, 9045-9050.

(14) Lund, A.; van der Velden, N. M.; Persson, N.-K.; Hamedi, M. M.; Müller, C. Electrically Conducting Fibres For E-Textiles: An Open Playground for Conjugated Polymers and Carbon Nanomaterials. Mater. Sci. Eng. R Rep. 2018, 126, 1-29.

(15) Kroon, R.; Mengistie, D. A.; Kiefer, D.; Hynynen, J.; Ryan, J. D.; Yu, L.; Müller, C. Thermoelectric Plastics: From Design to Synthesis, Processing and Structure-Property Relationships. Chem. Soc. Rev. 2016, 45, 6147-6164.

(16) Russ, B.; Glaudell, A.; Urban, J. J.; Chabinyc, M. L.; Segalman, R. A. Organic Thermoelectric Materials for Energy Harvesting and Temperature Control. Nature Rev. Mater. 2016, 1, 16050.

(17) Blackburn, J. L.; Ferguson, A. J.; Cho, C.; Grunlan, J. C. CarbonNanotube-Based Thermoelectric Materials and Devices. Adv. Mater. 2018, 30, 1704386.

(18) Li, P.; Guo, Y.; Mu, J. K.; Wang, H. Z.; Zhang, Q. H.; Li, Y. G. Single-Walled Carbon Nanotubes/Polyaniline-Coated Polyester Thermoelectric Textile with Good Interface Stability Prepared by Ultrasonic Induction. RSC Adv. 2016, 6, 90347-90353.

(19) Ma, W. G.; Liu, Y. J.; Yan, S.; Miao, T. T.; Shi, S. Y.; Xu, Z.; Zhang, X.; Gao, C. Chemically Doped Macroscopic Graphene Fibers with Significantly Enhanced Thermoelectric Properties. Nano Res. 2018, 11, 741-750.

(20) Ito, M.; Koizumi, T.; Kojima, H.; Saito, T.; Nakamura, M. From Materials to Device Design of a Thermoelectric Fabric for Wearable Energy Harvesters. J. Mater. Chem. A 2017, 5, 12068-12072.

(21) Kirihara, K.; Wei, Q.; Mukaida, M.; Ishida, T. Thermoelectric Power Generation Using Nonwoven Fabric Module Impregnated with Conducting Polymer PEDOT:PSS. Synth. Met. 2017, 225, 41-48.

(22) Cho, C.; Wallace, K. L.; Tzeng, P.; Hsu, J. H.; Yu, C.; Grunlan, J. C. Outstanding Low Temperature Thermoelectric Power Factor from Completely Organic Thin Films Enabled by Multidimensional Conjugated Nanomaterials. Adv. Energy Mater. 2016, 6, 1502168.

(23) Sarabia-Riquelme, R.; Craddock, J.; Morris, E. A.; Eaton, D.; Andrews, R.; Anthony, J.; Weisenberger, M. C. Simple, Low-Cost, Water-Processable n-Type Thermoelectric Composite Films from Multiwall Carbon Nanotubes in Polyvinylpyrrolidone. Synth. Met. 2017, 225, 86-92.

(24) Kim, S. L.; Choi, K.; Tazebay, A.; Yu, C. Flexible Power Fabrics Made of Carbon Nanotubes for Harvesting Thermoelectricity. ACS Nano 2014, 8, 2377-2386.

(25) Yu, C. H.; Murali, A.; Choi, K. W.; Ryu, Y. Air-Stable Fabric Thermoelectric Modules Made of $\mathrm{n}$ - and $\mathrm{p}$-Type Carbon Nanotubes. Energy Environ. Sci. 2012, 5, 9481-9486.

(26) Aikawa, S.; Kim, S.; Thurakitseree, T.; Einarsson, E.; Inoue, T.; Chiashi, S.; Tsukagoshi, K.; Maruyama, S. Carrier Polarity Engineering in Carbon Nanotube Field-Effect Transistors by Induced Charges in Polymer Insulator. Appl. Phys. Lett. 2018, 112, 013501.

(27) Hewitt, C. A.; Kaiser, A. B.; Roth, S.; Craps, M.; Czerw, R.; Carroll, D. L. Multilayered Carbon Nanotube/Polymer Composite Based Thermoelectric Fabrics. Nano Lett. 2012, 12, 1307-1310.

(28) Luo, J.; Cerretti, G.; Krause, B.; Zhang, L.; Otto, T.; Jenschke, W.; Ullrich, M.; Tremel, W.; Voit, B.; Pötschke, P. PolypropyleneBased Melt Mixed Composites with Singlewalled Carbon Nanotubes for Thermoelectric Applications: Switching From p-Type to n-Type by the Addition of Polyethylene Glycol. Polymer 2017, 108, 513-520.

(29) Dörling, B.; Ryan, J. D.; Craddock, J. D.; Sorrentino, A.; El Basaty, A.; Gomez, A.; Garriga, M.; Pereiro, E.; Anthony, J. E.; Weisenberger, M. C.; Goni, A. R.; Müller, C.; Campoy-Quiles, M. Photoinduced p- to $\mathrm{n}$-Type Switching in Thermoelectric PolymerCarbon Nanotube Composites. Adv. Mater. 2016, 28, 2782-2789.

(30) Kingston, C.; Zepp, R.; Andrady, A.; Boverhof, D.; Fehir, R.; Hawkins, D.; Roberts, J.; Sayre, P.; Shelton, B.; Sultan, Y.; Vejins, V.; Wohlleben, W. Release Characteristics of Selected Carbon Nanotube Polymer Composites. Carbon 2014, 68, 33-57. 
(31) Köhler, A. R.; Som, C.; Helland, A.; Gottschalk, F. Studying the Potential Release of Carbon Nanotubes Throughout the Application Life Cycle. J. Cleaner Prod. 2008, 16, 927-937.

(32) Steven, E.; Saleh, W. R.; Lebedev, V.; Acquah, S. F. A.; Laukhin, V.; Alamo, R. G.; Brooks, J. S. Carbon Nanotubes on a Spider Silk Scaffold. Nat. Commun. 2013, 4, 2435.

(33) Andrews, R.; Jacques, D.; Qian, D. L.; Rantell, T. Multiwall Carbon Nanotubes: Synthesis and Application. Acc. Chem. Res. 2002,

35, 1008-1017.

(34) Jacques, D.; Andrews, R. U.S. Patent 7160531 B1, 2007.

(35) Jacques, D.; Andrews, R., U.S. Patent 7504078 B1, 2009. 